\title{
Approximating Power System Dynamics and Energy Functions by Quasi-Gradient Models
}

\author{
Christopher L. DeMarco \\ Department of Electrical and Computer Engineering \\ University of Wisconsin-Madison \\ 1415 Johnson Drive \\ Madison, WI 53706 USA
}

\section{ABSTRACT}

Power systems models that possess a well defined Lyapunov function frequently share the following structure: the normal form of their system state equations may be expressed as a constant matrix multiplying the gradient of a scalar function of state. This structure has previously been labelled as "quasi-gradient," and the scalar function shown to be a natural Lyapunov function for the system. Unfortunately, as detailed load/network representations or control loops are added to the model, the quasi-gradient structure is often lost. This paper builds on previous work by proposing a new approach to defining energy functions for models lacking the quasi-gradient structure. A modified Lyapunov function is derived for the quasi-gradient with the property that its derivative along trajectories of the system is a strictly negative definite quadratic form. This in tum guarantees that the property of the Lyapunov function being non-increasing along trajectories is preserved for certain perturbations in the vector field. In particular, this property is shown to be preserved under the condition that transfer conductances are perturbed within a sufficiently small ball about zero.

\section{INTRODUCTION AND BACKGROUND}

This paper will extend the work of [1] by examining those cases where the specialized form of the power system dynamics ("quasi-gradient structure") can no longer be applied. For example, consider the perennial problem of applying Lyapunov's direct method in power systems models that include resistive terms ("transfer conductances") in their transmission line models. In the vocabulary of [1], this problem manifests itself in the fact that the differential equations modeling the system with transfer conductances do not have the quasi-gradient structure. However, the premise of this paper is that the quasi-gradient structure still lends some insight into construction of suitable Lyapunov functions for the new system.

The approach is to find a quasi-gradient model that approximates the dynamics of the more detailed model. A modified Lyapunov function is derived for the quasi-gradient with the property that its derivative along trajectories of the system is a strictly negative definite quadratic form. This in tum guarantees that the property of the Lyapunov function being non-increasing dong trajectories is preserved for certain perturbations in the rector field (i.e., the Lyapunov function obtained ensures rotus stabiliby). In particular. this property is shown to he preserver under the condition that transfer conductances are perturbed within a sufficiently small ball about zero.
As a consequence of this approach some of the convenient properties usually associated with Lyapunov functions for power system models will be sacrificed. For example, in our construction it will no longer be true that the gradient of the Lyapunov function is zero at all equilibria; only the stable equilibrium will have this property. Still, the new construction will yield a valid Lyapunov function that is locally positive definite about the equilibrium of interest, and non-increasing along system trajectories near this equilibrium. While the current state of the analysis only guarantees the latter property in a neighborhood of the equilibrium of interest, it is hoped that this construction will prove useful for examining non-local analysis, such as estimating the region of attraction.

\section{REVIEW OF QUASI-GRADIENT DYNAMICAL} SYSTEM STRUCTURE

The results described in [1] suggested consideration of systems of the form

$$
\dot{\mathbf{x}}=-\mathrm{A} \nabla \mathrm{V}\left(\mathbf{x}, \mathbf{x}^{0}\right)
$$

where:

$$
\begin{aligned}
& V: \mathbf{R}^{\mathrm{n}} \times \mathbf{R}^{\mathrm{n}} \rightarrow \mathbf{R} \text { is continuously differentiable, } \\
& \mathbf{A} \in \mathbf{R}^{\mathrm{n} \times \mathrm{n}}, \operatorname{det}(\mathbf{A}) \neq 0, \\
& V\left(\mathbf{x}, \mathbf{x}^{0}\right)=0 \text { at } \mathbf{x}=\mathbf{x}^{0},
\end{aligned}
$$

and

$$
\nabla V\left(\mathbf{x}, \mathbf{x}^{0}\right)=\mathbf{0} \text { at } \mathbf{x}=\mathbf{x}^{0}
$$

A key assumption in [1] that allows $\mathrm{V}\left(\mathbf{x}^{0}, \mathbf{x}^{0}\right)$ to serve as a Lyapunov function relates the structure of $A, \nabla V\left(x^{0}, x^{0}\right)$, and trajectories of (1). Let

$$
S=\left\{\mathbf{x} \mid \nabla V\left(\mathbf{x}, \mathbf{x}^{0}\right)^{\mathrm{T}}\left(\mathbf{A}+\mathbf{A}^{\mathrm{T}}\right) \nabla V\left(\mathbf{x}, \mathbf{x}^{0}\right)=0\right\}
$$

Assumption 1:

The matrix $\left(\mathbf{A}+\mathbf{A}^{\mathrm{T}}\right)$ is positive semi-definite. Furthermore, the only complete trajectories of (1) contained in $S$ are of the form $x(t)=x^{E}$ for all $t \in[0, \infty)$. where $\nabla V\left(x^{\mathbb{E}}, \mathbf{x}^{0}\right)=0$.

ISCAS '89

CH2692-2/89/0000-1966 \$1.00 (c) 1989 IEEE 
Note that the second condition in assumption 1 is trivially satisfied if $\left(\mathbf{A}+\mathbf{A}^{\mathrm{T}}\right)$ is strictly positive definite. However, for almost ail power system examples of interest, $\left(\mathbf{A}+\mathbf{A}^{\mathrm{T}}\right)$ is rank degenerate, while $\mathbf{A}$ itself is full rank. Therefore the second condition is important. Systems of the form described in (1) and satisfying assumption 1 will be termed "quasi-gradient."

In (1), the dependence of $\mathrm{V}\left(\mathbf{x}, \mathrm{x}^{0}\right)$ on the equilibrium $x^{0}$ is shown explicitly. However, in most Lyapunov analyses for power systems, this quantity will be fixed at the outset of the study. Therefore, in the following discussion the dependence of the Lyapunov function on the equilibrium will be suppressed in our notation; i.e., the second argument as understood in (1) will not $b$ displayed. However, in parts of our discussion it will prove convenient to partition the state vector $\mathbf{x}$ into several components. Hence, the Lyapunov functions shown below may have several arguments; these should be understood as components of $\mathbf{x}$

In [1], four types of power system models were examined to contirm that they did indeed possess quasi-gradient structure. The simplest was the model taken from the classic work of El-Abiad [2]. This model will be used to illustrate the ideas to be developed here, though the approach is directly applicable to any of the power system models cataloged in [1]. In standard state variable form, the system dynamics for the El-Abiad model are given by:

$$
\begin{aligned}
& \delta=\omega \\
& \omega=-M^{-1}(\mathbf{D} \omega+\mathbf{f}(\delta)) \\
& \mathbf{M}=\operatorname{diag}\left(\mathbf{M}_{1}, \mathbf{M}_{2}, \ldots, \mathbf{M}_{\mathrm{n}}\right) \\
& \mathbf{D}=\operatorname{diag}\left(\mathrm{D}_{1}, \mathrm{D}_{2}, \ldots, \mathrm{D}_{\mathrm{n}}\right)
\end{aligned}
$$$$
\text { where }
$$

and

$$
f_{i}(\delta)=E_{i}^{2} G_{i i}-P_{i}+\sum_{j=1, j \neq i}^{n} E_{i} E_{j} B_{i j} \sin \left(\delta_{i}-\delta_{j}\right)
$$

The associated Lyapunov function for the system will be taken as thut defined in [2], given by:

$$
\begin{aligned}
& V(\omega, \delta)=\sum_{k=1}^{n} \frac{1}{2} \mathbf{M}_{k} \omega_{k}^{2}+\left(E_{k}^{2} G_{k k}-P_{k}\right)\left(\delta_{k}-\delta_{k}^{0}\right) \\
& +\sum_{k=1}^{n} \sum_{j=k+1}^{n} E_{k} E_{j} B_{k j}\left[\cos \left(\delta_{k}^{0}-\delta_{j}^{0}\right)-\cos \left(\delta_{k}-\delta_{j}\right)\right]
\end{aligned}
$$

The state vector is partitioned and ordered as $\left(\omega^{\mathrm{T}}, \delta^{\mathrm{T}}\right)^{\mathrm{T}}$. With this convention, the dynamics of (3) take the forro described in (1) if $\mathbf{A}$ is chosen as:

$$
\mathbf{A}=\left[\begin{array}{cc}
\mathbf{M}^{-1} \mathbf{D} \mathbf{M}^{-1} & \mathbf{M}^{-1} \\
-\mathbf{M}^{-1} & \mathbf{0}
\end{array}\right]
$$

Note that if $\mathrm{V}(\omega, \delta)$ is chosen as the candidate Lyapunov function, the derivative with respect to time along trajectories of (1) is a negative semi-definite quadratic form determined by the symmetric part of $A$, i.e. $1 / 2\left(A+A^{T}\right)$.
Consider now what happens when transfer conductances are added to this model. The effect is simply to modify the components of the function $f$. In particular, replace $f$ in (3) with a new function $h$ having components:

$$
\begin{aligned}
h_{i}(\delta)= & E_{i}^{2} G_{i i}-P_{i}+\sum_{j=1, j \neq i}^{n} E_{i} E_{j} B_{i j} \sin \left(\delta_{i}-\delta_{j}\right) \\
& +\sum_{j=1}^{n} E_{i} E_{j} G_{i j} \cos \left(\delta_{i}-\delta_{j}\right)
\end{aligned}
$$

This yields the new dynamics:

$$
\begin{aligned}
& \dot{\delta}=\omega \\
& \dot{\omega}=-\mathrm{M}^{-1}(\mathrm{D} \omega+\mathrm{h}(\delta))
\end{aligned}
$$

The basic problem which prevents (7) from possessing quasigradient structure, and hence an easily identified Lyapunov function, is the fact that $\mathbf{h}(\delta)$ above is no longer an exact function [5]. This is easily verified by examining the Jacobian of $h$ with respect to $\delta$; the resulting matrix function is not symmetric. A naive proposal to solve this problem would be simply to use the $V$ function as defined for the original system as a candidate Lyapunov function for the new system with transfer conductances. Two difficulties arise in attempting this approach.

i) The derivative of $\mathrm{V}$ along trajectories of the new system can no longer be guaranteed non-positive, even in a small neighborhood of the equilibrium point of interest.

ii) The addition of nonzero transfer conductances changes the location of the equilibrium, while $\mathrm{V}$ is unchanged. Hence the original $\mathrm{V}$ is no longer locally positive definite about the operating - oint.

To address these problems, the original $\mathrm{V}$ function will be modified in two steps. The first of these examines the sign definiteness of $\dot{V}$. It should be clear that part of the problem arises from the fact that even for the original system, $V$ as chosen results in an expression for $\dot{V}$ that is only negative semi-definite. This leaves no "margin of robustness" in the analysis when the $f$ function is perturbed to $h$, even for arbitrarily small perturbations. The first step below yields a Lyapunov function for the original system whose derivative along trajectories is a strictly negative definite form.

Consider the following function $U(\omega, \delta)$ :

$$
\begin{aligned}
& U(\omega, \delta)=\sum_{k=1}^{n} \frac{1}{2} M_{k} \omega_{k}^{2}+\left(E_{k}^{2} G_{k k}-P_{k}\right)\left(\delta_{k}-\delta_{k}^{0}\right) \\
& +\sum_{k=1}^{n} \sum_{j=k+1}^{n} E_{k} E_{j} B_{k j}\left[\cos \left(\delta_{k}^{0}-\delta_{j}^{0}\right)-\cos \left(\delta_{k}-\delta_{j}\right)\right] \\
& +\mu f^{T}(\delta) \omega
\end{aligned}
$$


Here $\mu$ is a positive scalar to be specified later. Evaluating the derivative of $U$ along trajectories of the original system (3), one obtains:

$$
\begin{aligned}
\frac{\partial U}{\partial \mathbf{x}} \dot{\mathbf{x}} & =\left\{\frac{\partial \mathrm{V}}{\partial \mathbf{x}}+\mu\left[\mathbf{f}^{\mathrm{T}}(\delta) \mid \omega^{\mathrm{T}} \frac{\partial \mathbf{f}}{\partial \delta}\right]\right\}\left[\begin{array}{cc}
\mathbf{M}^{-1} \mathbf{D} \mathbf{M}^{-1} & \mathbf{M}^{-1} \\
-\mathbf{M}^{-1} & 0
\end{array}\right]\left[\begin{array}{l}
\mathbf{M} \omega \\
\mathbf{f}(\delta)
\end{array}\right] \\
& =-\left[\omega^{\mathrm{T}} \mathbf{f}^{\mathrm{T}}(\delta)\left[\begin{array}{cc}
\left(\mathbf{D}-\mu \frac{\partial \mathbf{f}}{\partial \delta}\right) & 1 / 2 \mu \mathbf{M}^{-1} \mathbf{D} \\
1 / 2 \mu \mathbf{M}^{-1} \mathbf{D} & \mu \mathbf{M}^{-1}
\end{array}\right]\left[\begin{array}{c}
\omega \\
\mathbf{f}(\delta)
\end{array}\right]\right.
\end{aligned}
$$

Denote the matrix defining the quadratic form above as

$$
\mathbf{N}_{\mu}(\delta)=\left[\begin{array}{cc}
\left(\mathbf{D}-\mu \frac{\partial \mathbf{f}}{\partial \delta}\right) & 1 / 2 \mu \mathbf{M}^{-1} \mathbf{D} \\
1 / 2 \mu \mathbf{M}^{-1} \mathbf{D} & \mu \mathbf{M}^{-1}
\end{array}\right]
$$

In expressing $\mathbf{N}_{\mu}(\delta)$ in the form shown above, we have taken advantage of the fact that $\mathbf{M}$ and $\mathbf{D}$ are diagonal matrices, and therefore commute, and that $\partial \mathbf{r} / \partial \delta$ is symmetric, and therefore is equal to its transpose. The following two inequalities offer a simple sufficiency test to guarantee $\mathbf{N}_{\mu}(\boldsymbol{\delta})$ positive definite. (Note that since all of the four sub-blocks composing $N_{\mu}(\delta)$ are symmetric real matrices, they will possess purely real eigenvalues.)

$$
\begin{gathered}
\lambda_{\text {min }}\left(\mathbf{D}-\mu \frac{\partial \mathbf{f}}{\partial \delta}\right)>0 \\
\mu \lambda_{\min }\left(\mathbf{D}-\mu \frac{\partial \mathbf{f}}{\partial \delta}\right) \lambda_{\text {min }}\left(\mathbf{M}^{-1}\right)>\frac{\mu^{2}}{4}\left(\lambda_{\text {min }}\left(\mathbf{M}^{-1} \mathbf{D}\right)\right)^{2}
\end{gathered}
$$

The largest singular value of $\partial \mathbf{f} / \partial \delta$ can be uniformly bounded above by a finite scalar easily computable from knowledge of the bus admittance matrix for the network. It follows that there must exist a positive value of $\mu_{\max }$ such that $N_{\mu}(\delta)$ is guaranteed iniformly (over $\delta$ ) positive definite for $0<\mu<\mu_{\max }$. For $\mu$ in this range the derivative of $U(\omega, \delta)$ along trajectories of $(3)$ is Itlaranteed to be a strictly negative definite quadratic form.

Next, we wish to demonstrate that $U$ must be positive definite bout any equilibrium that has a stable linearization. These steps follow the reasoning described in [1]. Summarizing this briefly, $\because 1$ observe that at an equilibrium point of (3), the gradient of $U$

$\cdots 1$ be zero. It follows that the only nonzero terms in a Taylor $\therefore$. nsion of $U$ about an equilibrium of (3) are of second order or We can conclude that $\nabla^{2} \mathrm{U}\left(\mathbf{x}^{0}\right)$ must therefore be positive definite. If it were not, there would exist a point $\widetilde{\mathbf{x}}$ arbitrarily close to $x^{0}$ with the property that $U(\widetilde{\mathbf{x}})<0$. Since $U(\mathbf{x}(t))$ is nonincreasing along trajectories, solutions of (3) originating at $\widetilde{\mathbf{x}}$ never return to $x^{0}$. This would contradict the stability of the linearization.

Our first problem is therefore eliminated: we have a Lyapunov function that yields a strictly negative definite quadratic form for its derivative along trajectories of the original system (3). Our second problem of a change in the equilibrium is also dealt with easily. Let $\mathbf{x}^{0}=\left(0, \delta^{0}\right)$ be the equilibrium point of interest for (7).
Define $g(\delta)$ as the difference between $f(\delta)$ and $h(\delta)$ due to the transfer conductances, i.e.

$$
g_{i}(\delta)=\sum_{j=1}^{n} E_{i \neq j} E_{j} G_{i j} \cos \left(\delta_{i}-\delta_{j}\right)
$$

Let $\Delta \mathbf{P}$ represent the value of this difference at the equilibrium,

$$
\Delta \mathbf{P}=\mathbf{g}\left(\delta^{0}\right)
$$

Finally, let

$$
\tilde{f}(\delta)=f(\delta)+\Delta \mathbf{P}
$$

and

$$
\Delta \mathbf{f}(\delta)=g(\delta)-\Delta \mathbf{P}
$$

With this construction, it follows that:

$$
\begin{aligned}
& h(\delta)=\mathfrak{f}(\delta)+\Delta f(\delta), \\
& \mathbf{f}\left(\delta^{0}\right)=0, \text { and } \\
& \Delta f\left(\delta^{0}\right)=0 .
\end{aligned}
$$

Now, modify the $U(\omega, \delta)$ as defined in (8) by replacing $P$ by $\mathbf{P}+\Delta \mathbf{P}$. The Lyapunov function candidate for $(7)$ becomes

$$
\begin{aligned}
& W(\omega, \delta)=\sum_{k=1}^{n} \frac{1}{2} M_{k} \omega_{k}^{2}+\left(E_{k}^{2} G_{k k}-P_{k}-\Delta P_{k}\right)\left(\delta_{k}-j\right. \\
& +\sum_{k=1}^{n} \sum_{j=k+1}^{n} E_{k} E_{j} B_{k j}\left[\cos \left(\delta_{k}^{0}-\delta_{j}^{0}\right)-\cos \left(\delta_{k}-\delta_{j}\right)\right] \\
& +\mu f^{T}(\delta) \omega
\end{aligned}
$$

Clearly $\mathrm{W}\left(0, \delta^{0}\right)=0$, more importantly, $\nabla \mathrm{W}\left(0, \delta^{0}\right)=\mathbf{0}$, so we at least satisfy the necessary condition for $W$ to have a local minimum at the equilibrium point of interest. Evaluating the time derivative of $\mathrm{W}$ along trajectories of (7) yields

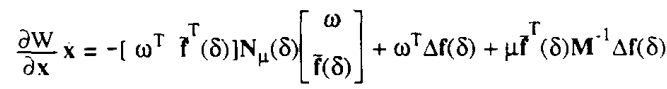

To express this quantity in a more convenient form, define the matrices $[f]$ and $[\Delta f]$ by placing the elements of $\boldsymbol{f}$ and $\Delta f$ on their respective diagonals:

$$
[\tilde{f}]=\operatorname{diag}\left(\tilde{f_{1}}, \tilde{\tilde{f}_{2}}, \ldots, \tilde{f_{n}}\right\}
$$

ind

$$
[\Delta \mathbf{f}]=\operatorname{diag}\left\{\Delta \mathrm{f}_{1}, \Delta \mathrm{f}_{2}, \ldots, \Delta \mathrm{f}_{\mathrm{n}}\right\} .
$$


The second and third terms on the right hand side of (18) may be expressed as

$$
\left.\left[\begin{array}{ll}
\omega^{T} & \left.\mathbf{f}^{T}(\delta)\right]
\end{array}\right] \begin{array}{cc}
0 & 1 / 2\left[\mathbf{f}^{-1}[\Delta f]\right. \\
1 / 2[\Delta f]\left[f^{-1}\right. & \mu \mathbf{M}^{-1}\left[\mathbf{f}^{-1}[\Delta f]\right.
\end{array}\right]\left[\begin{array}{c}
\omega \\
f(\delta)
\end{array}\right]
$$

Tir establish that (18) represents a negative definite quadratic form equires conditions under which $\mathrm{N}_{\mu}(\delta)$ will dominate the matrix defining the quadratic form in (21). Suppose $\gamma$ represents an upper bound on the magnitude of the $G_{i j}$ terms; i.e. $\left|G_{i j}\right| \leq \gamma$. Further suppose that the equilibrium of interest has the property that the Jacobian of $f(\delta)$ evaluated at $\delta^{0}$ is nonsingular. It follows that there must exist a neighborhood $\mathcal{N}$ (about $\delta^{0}$, with the property that $\left\|[T(\delta)]^{-1}[\Delta f(\delta)]\right\|$ can be bounded by a positive constant times $\gamma$ within $\mathcal{N}$ This insures that for $\gamma$ sufficiently small, the right hand side of (18) is a strictly negative definite quadratic form within that neighborhood.

\section{v. CONCLUSIONS}

This paper describes a method of constructing Lyapunov functions for quasi-gradient systems that insures the resulting derivative along trajectories will be a strictly negative definite yuadratic form in a neighborhood of the equilibrium. This in turn guarantees that the analysis will be robust with respect to smail changes in the differential equations defining the system model. In particular, it is shown that small transfer conductances can be added to the model. Under the assumption that the system with transfer conductances has a strictly stable linearization at the equilibrium point of interest, the new Lyapunov function remains locally positive definite and its derivative remains locally negative vefinite about this equilibrium

Bezause the new Lyapunov function is only guaranteed valid in a neighborhood of the equilibrium, one might question if a fixed yuladratic Lyapunov function obtained from linearizing the system model at that point might not yield similar results. Given the close relationship of the proposed Lyapunov to the nonlinear structure of the system dynamics, there is strong reason to believe that it will be valid over a much larger neighborhood than any fixed quadratic function of the form $\mathbf{x}^{\mathrm{T}} \mathbf{P x}$. Future research investigating this issue in more detail would be most useful.

\section{REFERENCES}

11] C. L. DeMarco "A New Method of Constructing Lyapunov Functions for Power Systems," Proc. 1988 IEEE Int. Symp. Circuits and Systems, pp. 905-908, Helsinki, Finland, June 1988.

12] A. H. El-Abiad and K. Nagappan, "Transient stability regions of multimachine power systems," IEEE Trans. Power App. and Sys., v. PAS-85, pp. 169-179, Feb. 1966

[3] N. Narasimhamurthi, "On the existance of energy functions for systems with transmission losses,".IEEE Trans. Circuits and Systems, vol. CAS-31, no. 2, pp. 199-203. Feb. 1984.
[4] H. G. Kwatny, L. Y. Bahar, A. K. Pasrija, "Energy-like Lyapunov functions for power system stability analysis,"IEEE Trans. Circuits and Systems, vol. CAS-32, no. 11, pp. $1140-1149$, Nov. 1985.

[5] W.H. Fleming, Functions of Several Variables, AddisonWesley, Reading, MA, 1965. 